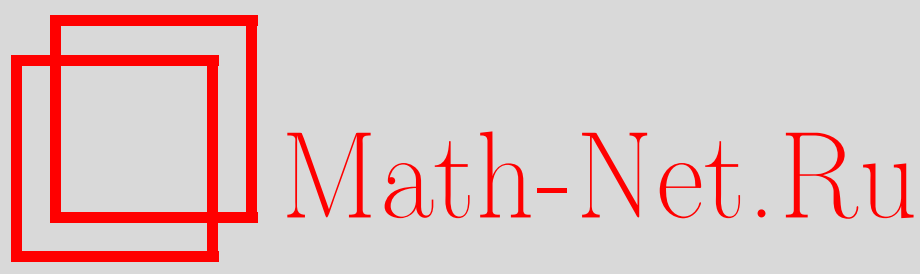

А. В. Угланов, Поверхностные интегралы в пространствах Фреше, Матем. сб., 1998, том 189, номер 11, 139-157

DOI: https://doi.org/10.4213/sm374

Использование Общероссийского математического портала Math-Net.Ru подразумевает, что вы прочитали и согласны с пользовательским соглашением

http: //www.mathnet.ru/rus/agreement

Параметры загрузки:

IP : 54.224 .60 .19

26 апреля 2023 г., 17:07:08 
УДК 517.98

\author{
А.В. Угланов
}

\title{
Поверхностные интегралы в пространствах Фреше
}

\author{
Строится теория интегрирования по гиперповерхностям в сепарабельных про- \\ странствах Фреше. Даются соответствующие определения и доказываются ос- \\ новные формулы теории: формула повторного интегрирования, интегрирования \\ по частям, Гаусса-Остроградского, Грина. \\ Библиографиял: 32 названия.
}

\section{Введение}

В ходе развития бесконечномерного анализа (в частности, дифференцирования и интегрирования на бесконечномерных пространствах) возник интерес математиков к интегралам по нелинейным бесконечномерным многообразиям. В рабо$\operatorname{tax}[1],[2]$ (видимо, пионерских) было построено сужение гауссовой меры на гиперповерхность и сформулирована теорема о дивергенции (формула Гаусса-Остроградского); при этом существенно использовалась гауссова специфика (см. еще [3]). А. В. Скороход [4], основываясь на понятии квазиинвариантности, ввел поверхностные вероятностные меры для негауссова случая. Использование идей дифференцирования мер позволило изучать знакопеременные (и даже векторные) поверхностные меры [5]-[7]. Ю. Л. Далецкий и Б. Д. Марянин начали рассмотрение мер на абстрактных многообразиях [8]. Из других исследований по теме отметим статьи $[9]-[13]$.

Подход, предложенньй в [5]-[7], оказался весьма плодотворным; на его основе удалось построить содержательную теорию поверхностного интегрирования в банаховых пространствах; эта теория нашла серьезные применения в таких областях, как бесконечномерные распределения и дифференциальные уравнения [5], [14]-[21], случайные процессы [7], [16], [22], приближение функций бесконечномерного аргумента [23], векторное интегрирование [24], вариационное исчисление на банаховых пространствах [25]. Однако после основополагающей работы [6] главное внимание уделялось именно приложениям теории; приложениям же посвящались почти все публикации: статья [7] - едва ли не единственное исключение. Между тем аппарат, предложенный в [5]-[7], имеет ряд узких мест, которые затрудняют или вообще сдерживают дальнейшие применения. К числу таких мест относятся следующие.

1. Интегрирование возможно лишш на поверхностях, гладких по всем направлениям основного пространства. Это ограничение не слишком сушественно для пространства с гладкой нормой, но становится непреодолимым при рассмотрении

Работа вьполнена при поддержке Российского фонда фундаментальных исследований (грант № 95-01-00604a). 
пространств, где крайне беден запас Фреше-дифференцируемых функций (в частности, винеровские пространства).

2. Краеугольным камнем всей конструкции [6] является теорема инвариантности. Однако в [6] теорема доказана в предположении двойной гладкости и рассматриваемых поверхностей, и генерируюшей объемной меры (для самого определения поверхностных мер требуется лишь однократная гладкость). Именно “благодаря" этому в статьях [7], [10], [15]-[21], [23] завышались требования гладкости соответствующих объектов, а в статьях [22], [23], [26] давались дополнительные разъяснения. Особенно неприятньм являлось требование двойной гладкости объемной меры: оно не позволяло заменять меру ее жордановыми составляющими (даже бесконечная гладкость меры гарантирует лиш однократную гладкость жордановых составляющих); во многих же случаях знакопостоянство генерирующей меры является решающим фактором (см.,например, доказательство теоремы 5 в настоящей работе).

3. Все рассмотрения касаются лишш банаховых многообразий (этот недостаток присуш почти всем известным автору исследованиям по поверхностному интегрированию).

Добавим еще, что для приложений первостепенное значение имеют формулы повторного интегрирования, Гаусса-Остроградского и Грина. Между тем эти формулы (для банаховых пространств) лишь анонсировались в [7], [22]; доказательства же до сих пор нигде не публиковались.

Настоящая работа устраняет указанные недостатки. Это уже дало положительные результаты: например, без интегрирования по негладким поверхностям были бы невозможны результаты работы [27].

Укажем еще следующее. Конструкция поверхностных мер и все результаты предлагаемой статьи распространяются на произвольные локально выпуклые линейные топологические пространства. Однако при этом все рассуждения (начиная уже с основных определений) резко усложняются и становятся доступными лишш узкому кругу специалистов по теории меры. Автор не пошел по этому пути, рассчитывая на читателей общематематического журнала.

\section{§1. Конструкция поверхностных мер}

Все встречающиеся в статье линейные пространства - действительные. Под мерой понимается действительнозначная счетноаддитивная функция, определенная на борелевской $\sigma$-алгебре $\Sigma_{X}$ метрического пространства $X$; совокупность всех таких мер обозначается через $M(X)$. Если $\mu \in M(X)$, то через $|\mu| \in M(X)$ обозначается полное изменение меры $\mu$. Для $\mu$-интегрируемой (т.е. $|\mu|$-интегрируемой) функции $f: X \rightarrow R^{1}$ через $f \mu$ обозначается мера на $X$, определяемая равенством $f_{\mu}(A)=\int_{A} f d \mu$. Дифференцируемость мер понимается в смысле [28] $\left(\tau_{s}\right.$-дифференцируемость). Иногда-это будет оговариваться-мерой будет назьваться счетноаддитивная функция $\Sigma_{X} \rightarrow[0, \infty]$. Подмножества метрических пространств рассматриваются, если не оговорено противное, с индуцированной метрикой.

Далее, $Z$ есть сепарабельное пространство $\Phi$ реше, $Z_{0}$ - линейно и непрерывно вложенное в $Z$ нормированное пространство, $Z_{0}^{*}$ - наделенное равномерной нормой сопряженное с $Z_{0}$ пространство, $\mu \in M(Z)$ - мера, дифференцируемая по всем 
направлениям из $Z_{0}$ (совокупность всех таких мер обозначим через $M^{1}$ ). Заметим, что если $\mu=\mu^{+}-\mu^{-}$есть жорданово разложение меры $\mu$, то $\mu^{ \pm} \in M^{1}$ $[28 ;$ гл. IV,$\S 2]$.

Пусть $X$ - замкнутое гиперподпространство $Z$, не содержащее $Z_{0}, a \in Z_{0} \backslash X, R_{a}$ - одномерное подпространство, порожденное вектором $a, A$-открытое (в $X)$ подмножество $X, f: A \rightarrow R_{a}$ - непрерывная функция, имеющая непрерьвную ограниченную производную $\Phi$ реше $f^{\prime}: A \rightarrow\left(X \cap Z_{0}\right)^{*}$ вдоль нормированного пространства $X \cap Z_{0}$ (сопряженное пространство $\left(X \cap Z_{0}\right)^{*}$ наделено равномерной нормой). Обозначим через $G=G(X, A, a, f)$ график (в произведении $X \times R_{a}=Z$ ) функции $f$ и для $Q \in \Sigma_{G}$ положим $T=T(Q)=\bigcup_{s \leqslant 0}\{Q+s a\}, \mu_{a}(Q)=D_{a} \mu(T)$ $\left(D_{a}\right.$ означает производную по направлению $a$ ). Так как функция $D_{a} \mu: \Sigma_{Z} \rightarrow R^{1}$ счетноаддитивна, то $\mu_{a}: \Sigma_{G} \rightarrow R^{1}$ есть мера на $G$; если $\mu \geqslant 0$, то и $\mu_{a} \geqslant 0$. Обозначим через $n=n(\omega) \in Z_{0}^{*}$ единичную нормаль к множеству $G$ в точке $\omega \in G$. Из предположений относительно $f$ вытекает, что функция $\omega \mapsto|(n(\omega), a)|^{-1}$ ограничена на $G$, так что корректно определена мера $\mu_{G}: \Sigma_{G} \rightarrow R^{1}$,

$$
\mu_{G}(Q)=\int_{Q}|(n, a)|^{-1} d \mu_{a}
$$

Сейчас наша главная цель - доказательство того, что мера $\mu_{G}$ полностью определяется мерой $\mu$ и множеством $G$ (т.е. не зависит от координатного представления $G=G(X, A, a, f))$.

Далее считаем, что $\|a\|=1$. Пусть $l: \Sigma_{R_{a}} \rightarrow[0, \infty]-$ мера Лебега на $R_{a}$, $l[0, a]=1$. Определим меру $|\mu|_{X}: \Sigma_{X} \rightarrow R^{1}$,

$$
|\mu|_{X}(E)=|\mu|\left(E+R_{a}\right) .
$$

Доказательства следующих лемм 1, 2 имеются в [29].

ЛЕмма 1. Справедливо неравенство

$$
|\mu| \leqslant\left|D_{a} \mu\right|_{X} \times l
$$

СЛЕДСТВИЕ. Мера $\mu$ абсолютно непрерьвна относительно меры $|\mu|_{X} \times l$ $\left(\mu \ll|\mu|_{X} \times l\right)$.

В силу соотношений $\mu \ll|\mu|_{X} \times l, D_{a} \mu \ll \mu$ (по поводу последнего см. [28; гл. 4, $\S 2])$ и теоремы Радона-Никодима существуют $|\mu|_{X} \times l$-интегрируемые функции $\varphi, \psi: Z=X \times R_{a} \rightarrow R^{1}$ такие, что $\mu=\varphi\left(|\mu|_{X} \times l\right), D_{a} \mu=\psi\left(|\mu|_{X} \times l\right)$. Далее принимается $z=x+y=(x, y), z \in Z, x \in X, y \in R_{a}$.

ЛЕмма 2. Для $|\mu|_{X} \times l$-почти всех $(x, y) \in Z$ справедливо равенство

$$
\varphi(x, y)=\int_{-\infty}^{y} \psi(x, t) d t .
$$

Далее считается, что функция $\varphi$ определена равенством (2); в силу леммы 2 равенство $\mu=\varphi\left(|\mu|_{X} \times l\right)$ сохраняется. Обозначим через $P_{X}^{a}$ проектор $Z$ на $X$, параллельный $a$. 
ЛЕмма 3. Для любого $Q \in \Sigma_{G}$ справедливо равенство

$$
\mu_{a}(Q)=\int_{P_{X}^{a} Q} \varphi(x, f(x)) d|\mu|_{X}(x) .
$$

ДокАЗАТЕЛЬСТво. Из определения, теоремы Фубини и равенства (2) имеем

$$
\begin{aligned}
\mu_{a}(Q)=D_{a} \mu(T) & =\int_{P_{X}^{a} Q} \int_{-\infty}^{f(x)} \psi(x, y) d y d|\mu|_{X}(x) \\
& =\int_{P_{X}^{a} Q} \varphi(x, f(x)) d|\mu|_{X}(x),
\end{aligned}
$$

что и требуется.

Пусть $Q \in \Sigma_{G}, \delta>0, \varepsilon \in[0, \delta] ; F: P_{X}^{a} Q \times[0, \delta] \rightarrow R^{a}$ - борелевская функция такая, что $F(x, 0) \equiv f(x), Q(\varepsilon)=\operatorname{gr} F(\cdot, \varepsilon) ; \Delta_{\varepsilon}: P_{X}^{a} Q \rightarrow R_{a}, \Delta_{\varepsilon}(x)=F(x, \varepsilon)-$ $f(x) ; Q_{\varepsilon}^{+}=\left\{\omega \in Q(\varepsilon): \Delta_{\varepsilon}\left(P_{X}^{a} \omega\right) \geqslant 0\right\}, Q_{\varepsilon}^{-}=Q(\varepsilon) \backslash Q_{\varepsilon}^{+} ; Q^{ \pm}=\left.\operatorname{gr} f\right|_{P_{X}^{a} Q_{\varepsilon}^{ \pm}}$, $T^{+}=T\left(Q_{\varepsilon}^{+}\right) \backslash T\left(Q^{+}\right), T^{-}=T\left(Q^{-}\right) \backslash T\left(Q_{\varepsilon}^{-}\right)$.

ЛЕмма 4. Предположим, ито при любом $x \in P_{X}^{a} Q$ функиия $F(x, \cdot):[0, \delta]$ $\rightarrow R_{a}$ дифферениируема, причем

$$
\sup _{x, \varepsilon}\left|\frac{d F}{d \varepsilon}(x, \varepsilon)\right|<\infty
$$

Тогда

$$
\lim _{\varepsilon \rightarrow 0} \varepsilon^{-1}\left[\mu\left(T^{+}\right)-\mu\left(T^{-}\right)\right]=\int_{Q} \frac{d F}{d \varepsilon}\left(P_{X}^{a} \omega, 0\right) d \mu_{a}(\omega)
$$

ДокАЗАТЕЛЬСТво. Из определений функции $\varphi$, множеств $T^{ \pm}$и теоремы Фубини получаем

$$
\mu\left(T^{+}\right)-\mu\left(T^{-}\right)=\int_{P_{X}^{a} Q} \int_{F(x, 0)}^{F(x, \varepsilon)} \varphi(x, y) d y d|\mu|_{X}(x)
$$

Из формул Лагранжа, леммы 2 и условия (3) получаем

$$
\begin{aligned}
\left|\frac{1}{\varepsilon} \int_{F(x, 0)}^{F(x, \varepsilon)} \varphi(x, y) d y\right| & =\left|\frac{d F}{d \varepsilon}(x, \theta \varepsilon) \varphi(x, F(x, 0)+\theta \varepsilon)\right| \\
& \leqslant c|\varphi(x, F(x, 0)+\theta \varepsilon)|=c\left|\int_{-\infty}^{F(x, 0)+\theta \varepsilon} \psi(x, y) d y\right| \\
& \leqslant c \int_{-\infty}^{\infty}|\psi(x, y)| d y \quad(\theta=\theta(x, \varepsilon) \in(0, a)) .
\end{aligned}
$$


$\mathrm{B}$ силу определения функции $\psi(x, y)$ и теоремы Фубини последняя функция $|\mu|_{X}$-интегрируема. Теперь, деля равенство (4) на $\varepsilon$, устремляя $\varepsilon$ к нулю, переходя к пределу под знаком внешнего интеграла и применяя лемму 3 , получим

$$
\begin{aligned}
\lim _{\varepsilon \rightarrow 0} \varepsilon^{-1}\left[\mu\left(T^{+}\right)-\mu\left(T^{-}\right)\right] & =\int_{P_{X}^{a} Q} \frac{d F}{d \varepsilon}(x, 0) \varphi(x, F(x, 0)) d|\mu|_{X}(x) \\
& =\int_{Q} \frac{d F}{d \varepsilon}\left(P_{X}^{a} \omega, 0\right) d \mu_{a}(\omega),
\end{aligned}
$$

что и требовалось.

Пусть есть еще одно представление $G=G(\bar{X}, \bar{A}, \bar{a}, f)\left(\bar{a} \in Z_{0} \backslash X,\|\bar{a}\|=1\right)$; тогда на $G$ определена еще одна мера $\bar{\mu}_{G}$.

Лемма 5. Справедливо равенство $\mu_{G}=\bar{\mu}_{G}$.

ДокАЗАТЕЛЬСтво. Так как $\mu_{G}=\left(\mu^{+}\right)_{G}-\left(\mu^{-}\right)_{G}$, то достаточно рассмотреть случай $\mu \geqslant 0$. Возьмем $N \in Z^{*}$ такой, что $N \neq 0,(N, X)=0$, и положим

$$
b=\bar{a}-(N, \bar{a})(N, a)^{-1} a .
$$

Пусть $X_{b}$ есть какое-то топологическое дополнение $R_{b}$ до пространства $X$. Рассмотрим класс

$$
K=\left\{A_{H, s, t} \subset A: A_{H, s, t}=\bigcup_{s<\tau<t}(H+\tau b), s, t \in R^{1}, s<t, H \text { открыто в } X_{b}\right\} .
$$

Далее считаем, что $X=\bar{X}$ (это не ограничивает общности: если $\bar{a} \in X$, то небольшим поворотом $X$ можно нарушить это включение) и для всех $\omega \in G$

$$
(n(\omega), a)>0, \quad(n(\omega), \bar{a})>0
$$

(эти функции от $\omega$ не меняют знак, так что предположение законно).

Фиксируем $Q: P_{X}^{a} Q \in K$. Рассмотрим функции

$$
\begin{array}{lll}
F(x, \varepsilon)=f(x)+\varepsilon(n(\omega), a)^{-1} a & \left(x \in P_{X}^{a} Q, \quad \omega=(x, f(x)), \quad \varepsilon \geqslant 0\right), \\
\bar{F}(x, \varepsilon)=\bar{f}(x)+\varepsilon(n(\omega), \bar{a})^{-1} \bar{a} & \left(x \in P_{X}^{\bar{a}} Q, \quad \omega=(x, \bar{f}(x)), \quad \varepsilon \geqslant 0\right) .
\end{array}
$$

В силу (6) выполняется равенство $T^{-}=\bar{T}^{-}=\varnothing$, так что по лемме 3

$$
\begin{aligned}
& \lim _{\varepsilon \rightarrow 0} \varepsilon^{-1} \mu\left(T^{+}\right)=\int_{Q}(n(\omega), a)^{-1} d \mu_{a}(\omega)=\mu_{G}(Q), \\
& \lim _{\varepsilon \rightarrow 0} \varepsilon^{-1} \mu\left(\bar{T}^{+}\right)=\int_{Q}(n(\omega), \bar{a})^{-1} d \mu_{\bar{a}}(\omega)=\bar{\mu}_{G}(Q) .
\end{aligned}
$$

Для $U \subset Z, x \in X$ положим $S_{x}^{a}(U)=\left\{y \in R_{a}: x+y \in U\right\}$ и обозначим через $d_{x}$ диаметр множества $S_{x}^{a}\left(T^{+} \Delta \bar{T}^{+}\right)$. Сейчас наша цель - доказательство соотношения $d_{x}=o(\varepsilon)(\varepsilon \rightarrow 0, x$ фиксировано). 
Для $z=(x, f(x))$ выберем точку $z+\Delta z \in Q$ так, чтобы

$$
z+\Delta z+\bar{k} \varepsilon \bar{a}=x+\lambda a,
$$

где $\bar{k}=(n(z+\Delta z), \bar{a})^{-1}, \lambda \in R^{1}$ (указанный выбор $z+\Delta z$ всегда возможен при достаточно малом $\varepsilon$; кроме того, $\Delta z \rightarrow 0$, если $\varepsilon \rightarrow 0)$. Проектируя равенство (9) на $X$ и $R_{a}$ и выражая $a$ из (5), получим

$$
\begin{gathered}
\Delta x+\bar{k} \varepsilon b=0 \\
f(x)+\Delta_{1} y+\bar{k} \varepsilon(N, \bar{a})(N, a)^{-1} a=\lambda a
\end{gathered}
$$

где $\Delta x=P_{X}^{a}(\Delta z), \Delta_{1} y=f(x+\Delta x)-f(x)$. Пусть $k=(n(z), a)^{-1}, \Delta y=k \varepsilon a$; тогда $d_{x}=\|f(x)+\Delta y-\lambda a\|$. Выражая $\lambda a$ из (11) и пользуясь формулой Лагранжа и равенством (10), находим

$$
\begin{aligned}
d_{x} & =\left\|\Delta y-\Delta_{1} y-\bar{k} \varepsilon(N, \bar{a})(N, a)^{-1} a\right\| \\
& =\left\|\Delta y-[f(x+\Delta x)-f(x)]-\bar{k} \varepsilon(N, \bar{a})(N, a)^{-1} a\right\| \\
& =\varepsilon\left|k+\left(f^{\prime}(x), b\right) \bar{k}-\bar{k}(N, \bar{a})(N, a)^{-1}+g(x, \Delta x)\right|,
\end{aligned}
$$

где функция $g(x, \Delta x)$ ограничена и стремится к нулю при $\Delta x \rightarrow 0$.

Запишем уравнение нормали $n(z)$ в координатах $\left(a^{0},\left(X \cap Z_{0}\right)^{0}\right)$, где $a^{0},\left(X \cap Z_{0}\right)^{0}$ суть ортогональные к $a, X \cap Z_{0}$ соответственно подпространства в $Z_{0}^{*}$. Имеем $n(z)=\left(-f^{\prime}(x), 1\right)\left\|\left(-f^{\prime}(x), 1\right)\right\|^{-1}$, где $f^{\prime}(x) \in a^{0}, 1 \in\left(X \cap Z_{0}\right)^{0}$, причем $(1, a)=1$.

Обозначив $c(x)=\left\|\left(-f^{\prime}(x), 1\right)\right\|^{-1}$, получим

$$
\begin{aligned}
(n, a)=c(x), \\
(n(z+\Delta z), \bar{a})=\left(n(z+\Delta z), b+(N, \bar{a})(N, a)^{-1} a\right) \\
=c(x+\Delta x)\left[-\left(f^{\prime}(x+\Delta x), b\right)+(N, \bar{a})(N, a)^{-1}\right] \\
=c(x)\left[-\left(f^{\prime}(x), b\right)+(N, \bar{a})(N, a)^{-1}\right]+g_{1}(x, \Delta x),
\end{aligned}
$$

где $g_{1}(x, \Delta x)$ - ограниченная функция и $g_{1}(x, \Delta x) \rightarrow 0$ при $\Delta x \rightarrow 0$. Подставляя полученные выражения в (12), имеем

$$
d_{x}=\varepsilon g_{2}(x, \Delta x)
$$

где $g_{2}(x, \Delta x)$ есть ограниченная функция и $g_{2}(x, \Delta x) \rightarrow 0$ при $\Delta x \rightarrow 0$.

Теперь положим $T=T^{+} \Delta \bar{T}^{+}$. В силу (1) и теоремы Фубини справедлива оценка

$$
\mu(T) \leqslant\left|D_{a} \mu\right|\left(T+R_{a}\right) \sup _{x \in P_{X}^{a} T} d_{x} .
$$

Пусть $\delta>0, \varepsilon_{n} \rightarrow 0, X^{n}=\left\{x \in X: d_{x}>\delta \varepsilon_{n}\right\}, \quad X_{m}=\bigcup_{n \geqslant m} X^{n}$, $X_{0}=\bigcap_{m \geqslant 1} X_{m}$. Так как $\lim _{\varepsilon \rightarrow 0} \Delta x=0$, см. (10), то из (13) и определения класса $K$ вытекает, что $\mu_{X}\left(X_{0}\right)=0$ (действительно, если $P_{X}^{a} Q=A_{H, s, t}$, то $X_{0} \subset(H+s b) \cup(H+t b)$, и остается воспользоваться дифференцируемостью 
меры $\mu_{X}$ по направлению $b$ ). Отсюда и из соотношения $\left|D_{a} \mu\right|_{X} \ll \mu_{X}$ получаем сушествование $m$ такого, что при $n \geqslant m$

$$
\left|D_{a} \mu\right|\left(X_{n}+R_{a}\right)<\delta
$$

В силу оценки (14), примененной к множествам $T \backslash\left(X_{n}+R_{a}\right)$ и $T \cap\left(X_{n}+R_{a}\right)$, учитывая равенство (13), неравенства (15) и $\sup _{x \in X \backslash X_{n}} d_{x} \leqslant \delta \varepsilon_{n}$, имеем для $n \geqslant m$

$$
\mu(T) \leqslant\left|D_{a} \mu\right|(Z) \delta \varepsilon_{n}+c_{2} \delta \varepsilon_{n}
$$

$\left(c_{2}-\right.$ константа, ограничивающая функцию $\left.g_{2}\right)$. Так как $\delta$ произвольно и $\varepsilon_{n} \rightarrow 0$, то $\lim _{\varepsilon \rightarrow 0} \varepsilon^{-1} \mu(T)=0$. Отсюда, из неравенства $\left|\mu\left(T^{+}\right)-\mu\left(\bar{T}^{+}\right)\right| \leqslant \mu(T)$ и равенств $(7),(8)$ вытекает, что $\mu_{G}(Q)=\bar{\mu}_{G}(Q)$.

Итак, на множествах из gr $\left.f\right|_{K}$ значения мер $\mu_{G}$ и $\bar{\mu}_{G}$ совпадают. Так как класс $K$ замкнут относительно конечных пересечений и образует базу топологии пространства $A$, то $\left.\operatorname{gr} f\right|_{K}$ замкнут относительно пересечений и образует базу топологии пространства $G\left(P_{X}^{a}\right.$ есть изоморфизм $G$ на $\left.A\right)$. Утверждение леммы теперь следует из сепарабельности $G$.

Рассмотрим два множества: $G=G(X, A, a, f)$ и $\bar{G}=G(\bar{X}, \bar{A}, \bar{a}, \bar{f})$; тогда на $G_{0}=G \cap \bar{G}$ определены две меры $\mu_{G}, \mu_{\bar{G}}$. Следующая теорема является основной в конструкции поверхностных мер.

ТЕОРема 1 (инвариантности). Справедливо равенство $\mu_{G}=\mu_{\bar{G}}$.

ДокАЗАТЕЛЬСтво. Опять считаем, что $\mu \geqslant 0$. Зафиксировав естественным образом направления нормалей $n, \bar{n}$, видим, что функции $n, \bar{n}: G_{0} \rightarrow Z_{0}^{*}$ непрерывны, так что справедливо включение $Q_{0}=\left\{\omega \in G_{0}: n(\omega)=\bar{n}(\omega)\right\} \in \Sigma_{G_{0}}$. Докажем сначала равенство $\mu_{G} \equiv \mu_{\bar{G}}$ на множестве $Q_{0}$. Из определения $Q_{0}$ и теоремы о неявной функции следует, что для любого $\omega \in Q_{0}$ существуют открытое множество $U(\omega) \ni P_{X}^{a} \omega$ в пространстве $X$ и непрерьвная функция $g: U(\omega) \rightarrow R_{a}$ с непрерьвной и ограниченной производной (вдоль пространства $X \cap Z_{0}$ ) такие, что $\bar{G} \cap\left\{U(\omega)+R_{a}\right\}=\operatorname{gr} g$. При этом, очеви дно, множество $U(\omega)$ можно выбрать так, что $U(\omega) \subset A$. Если теперь $Q \subset \operatorname{gr} g, Q \in \Sigma_{Q_{0}}$, то из леммы 5 (с заменой $G$ на $\operatorname{gr} g$ ), определения поверхностной меры и равенства $n(\omega)=\bar{n}(\omega)$ при $\omega \in Q$ имеем

$$
\mu_{\bar{G}}(Q)=\int_{Q}|(\bar{n}, \bar{a})|^{-1} d \mu_{\bar{a}}=\int_{Q}|(\bar{n}, a)|^{-1} d \mu_{a}=\int_{Q}|(n, a)|^{-1} d \mu_{a}=\mu_{G}(Q) .
$$

Итак, $\mu_{G} \equiv \mu_{\bar{G}}$ на $Q_{0}$ локально, а так как $Q_{0}$ сепарабельно, то $\mu_{G} \equiv \mu_{\bar{G}}$ на $Q_{0}$.

Для доказательства теоремы теперь достаточно убедиться в том, что $\mu_{G}\left(G_{0} \backslash Q_{0}\right)=\mu_{\bar{G}}\left(G_{0} \backslash Q_{0}\right)=0$. Считаем, что $X=\bar{X}$ (см. доказательство леммы 5 ), и пусть вектор $b$ определен равенством (5). Заметим, что включение $\omega_{0} \in G_{0}$ эквивалентно равенству

$$
(N, a)(N, \bar{a})^{-1} f\left(x_{0}\right)=\bar{f}\left(x_{0}-(N, a)(N, \bar{a})^{-1} f\left(x_{0}\right) b\right)
$$

$\left(x_{0}=P_{X}^{a} \omega_{0}, \omega_{0}=x_{0}+f\left(x_{0}\right) a\right.$, функции $f, \bar{f}$ считаются скалярами), а включение $\omega_{0} \in Q_{0}$ эквивалентно системе: (16) плюс равенство

$$
\begin{array}{r}
f^{\prime}\left(x_{0}\right)=(N, \bar{a})(N, a)^{-1} \bar{f}^{\prime}\left(x_{0}-(N, a)(N, \bar{a})^{-1} f\left(x_{0}\right) b\right) \\
-\left(\bar{f}^{\prime}\left(x_{0}-(N, a)(N, \bar{a})^{-1} f\left(x_{0}\right) b\right), b\right) f^{\prime}\left(x_{0}\right) .
\end{array}
$$


Рассмотрим функцию $F: A \rightarrow R^{1}$,

$$
F(x)=f(x)-\frac{(N, \bar{a})}{(N, a)} \bar{f}\left(x-\frac{(N, a)}{(N, \bar{a})} f(x) b\right) .
$$

Если $\omega_{0} \in G_{0} \backslash Q_{0}$, то из (16) следует, что $F\left(x_{0}\right)=0$, а из $(17)$ - что $F^{\prime}\left(x_{0}\right) \neq 0$. Возьмем вектор $d \in X \cap Z_{0}$ такой, что $\left(F^{\prime}\left(x_{0}\right), d\right) \neq 0$, и пусть $X_{d}$-некоторое топологическое дополнение $d$ до $X$. Из теоремы о неявной функции получаем существование открытых окрестностей $U\left(x_{0}\right), U_{d}\left(x_{0}\right)$ точек $x_{0}, P_{X_{d}}^{d} x_{0}\left(P_{X_{d}}^{d}\right.$ есть проектор $X$ на $X_{d}$, параллельный $d$; окрестности $U, U_{d}$ берутся в пространствах $X, X_{d}$ соответственно) и непрерывной функции $\varphi_{0}: U_{d}\left(x_{0}\right) \rightarrow R_{d}$ таких, что

$$
F^{-1}(0) \cap U\left(x_{0}\right)=\operatorname{gr} \varphi_{0}
$$

(график рассматривается в произведении $X_{d} \times R_{d}=X$ ). Рассмотрим множество $U\left(\omega_{0}\right)=\left\{\omega \in G_{0} \backslash Q_{0}: P_{X}^{a} \omega \in U\left(x_{0}\right)\right\} ; U\left(\omega_{0}\right)$ есть окрестность (в пространстве $\left.G_{0} \backslash Q_{0}\right)$ точки $\omega_{0}$. Из (16) и (18) получаем равенство $P_{X}^{a}\left[U\left(\omega_{0}\right)\right]=\operatorname{gr} \varphi_{0}$, откуда

$$
U\left(\omega_{0}\right) \subset\left\{\operatorname{gr} \varphi_{0}\right\}+R_{a} .
$$

Из дифференцируемости меры $\mu$ по направлению $d$ следует равенство

$$
\mu\left(\operatorname{gr} \varphi_{0}+R_{a}\right)=0,
$$

откуда $\left|D_{a} \mu\right|\left(\operatorname{gr} \varphi_{0}+R_{a}\right)=0$ и $\mu_{G}\left[G \cap\left(\operatorname{gr} \varphi_{0}+R_{a}\right)\right]=0$ (см. определение мер $\left.\mu_{a}, \mu_{G}\right)$. Включения $U\left(\omega_{0}\right) \subset G$ и (19) теперь дают равенство $\mu_{G}\left[U\left(\omega_{0}\right)\right]=0$. Так как пространство $G_{0} \backslash Q_{0}$ сепарабельно, то $\mu_{G}\left(G_{0} \backslash Q_{0}\right)=0$. В силу равноправия мер $\mu_{G}, \mu_{\bar{G}}$ имеем также равенство $\mu_{\bar{G}}\left(G_{0} \backslash Q_{0}\right)=0$, завершаюшее доказательство теоремы.

Назовем множество $\Omega \in \Sigma_{Z}$ простой поверхностью, если $\Omega \subset G(X, A, a, f)$ для некоторых $X, A, a, f ;$ поверхностью, если $\Omega$ есть счетное объединение простых поверхностей. Для поверхности $\Omega=\bigcup_{m=1}^{\infty} Q_{m}, Q_{m} \subset G_{m}, Q_{i} \cap Q_{j}=\varnothing$, определим функции $\mu_{\Omega}^{ \pm}: \Sigma_{\Omega} \rightarrow[0, \infty]: Q \mapsto \sum_{m=1}^{\infty}\left(\mu^{ \pm}\right)_{G_{m}}\left(Q \cap Q_{m}\right)$. Из теоремы 1 следует, что функции $\mu_{\Omega}^{ \pm}$корректно определены (т.е. не зависят от представления $\left.\Omega=\bigcup Q_{m}\right)$ и счетноаддитивны, так что корректно определена $\sigma$-конечная поверхностная мера $\mu_{\Omega}=\mu_{\Omega}^{+}-\mu_{\Omega}^{-}$.

ТЕОРема 2. Справедливы равенства $\left|\mu_{\Omega}\right|=|\mu|_{\Omega}, \mu_{\Omega}^{ \pm}=\left(\mu^{ \pm}\right)_{\Omega}$.

ДоКАЗАТЕЛЬСТво. Если $\Omega=G=G(X, A, a, f), Q \subset G$, то в силу теоремы 1 и леммы 3

$$
\left|\mu_{\Omega}\right|(Q)=\int_{P_{X}^{a}(Q)}|(n(x, f(x)), a)|^{-1}|\varphi(x, f(x))| d|\mu|_{X}(x) .
$$

Учитывая равенство $|\mu|=|\varphi|\left(|\mu|_{X} \times l\right)$ и опять применяя лемму 3 (с заменой $\mu$ на $|\mu|)$, находим, что $\left|\mu_{\Omega}\right|=|\mu|_{\Omega}$. Отсюда выводится, что это равенство справедливо и для общих поверхностей $\Omega$. Второе утверждение теоремы вытекает из первого с учетом соотношений $\mu^{ \pm}=\frac{1}{2}[|\mu| \pm \mu]$ и линейности соответствия $\mu \mapsto \mu_{\Omega}$.

Борелевская функция $\varphi: \Omega \rightarrow R^{1}$ называется $\mu_{\Omega}$-интегрируемой, если существуют интегралы $\int_{\Omega}|\varphi| d \mu_{\Omega}^{ \pm} ;$в этом случае полагается

$$
\int_{\Omega} \varphi d \mu_{\Omega}=\int_{\Omega} \varphi d \mu_{\Omega}^{+}-\int_{\Omega} \varphi d \mu_{\Omega}^{-}
$$


ТЕОРема 3. Отображсние $(\varphi, \mu) \mapsto \int_{\Omega} \varphi d \mu_{\Omega}$ билинейно на своей области определения.

ДокАЗАТЕЛЬСтво. Линейность по $\varphi$ есть общее свойство интеграла; линейность по $\mu$ вытекает из линейности соответствия $\mu \mapsto \mu_{\Omega}$.

ТЕОРема 4. Пусть функиия $u: Z \rightarrow R^{1}$ непрерывна, ограничена и ограниченно дифференцируема вдоль пространства $Z_{0}$. Тогда $u \mu \in M^{1} u(u \mu)_{\Omega}=u \mu_{\Omega}$ на $\Sigma_{\Omega}$.

ДокАЗАТЕЛьство. Легко видеть, что $D_{a}(u \mu)=D_{a} u \mu+u D_{a} \mu$, так что $u \mu \in M^{1}$. Пусть $G=G(X, A, a, f)$. Из предположений теоремы вытекает, что для любого $x \in X$ функция $u(x, \cdot): R_{a} \rightarrow R^{1}$ абсолютно непрерьвна, так что в силу лемм 2,3

$$
\begin{aligned}
u \mu & =(u \varphi)\left(|\mu|_{X} \times l\right), \\
(u \mu)_{a}(Q) & =\int_{P_{X}^{a} Q}(u \varphi)(x, f(x)) d|\mu|_{X}(x),
\end{aligned}
$$

$Q \in \Sigma_{G}$, откуда $(u \mu)_{a}=u \mu_{a}$ и $(u \mu)_{G}=u \mu_{G}$. Дальнейшее ясно.

\section{§ 2. Формула повторного интегрирования}

Обозначения: $V$ - открытое подмножество $Z ; C^{1}(V)$ - множество непрерывных действительных функций на $V$, имеющих непрерывную производную Фреше вдоль пространства $Z_{0} ; F \in C^{1}(V) ; V_{t}=\{z \in V: F(z)<t\} ; \Omega_{t}=\{z \in V: F(z)=t\}$ $\left(t \in R^{1}\right)$. Для любого $t \in R^{1}$ предполагаем, что $\Omega_{t}$ есть поверхность. Целью настоящего параграфа является доказательство следующего утверждения.

ТЕорема 5 (формула повторного интегрирования). Пусть $|\mu|\{z \in V$ : $\left.F^{\prime}(z)=0\right\}=0$, функция $\varphi: V \rightarrow R^{1} \quad \mu$-интегрируема. Тогда корректно и справедливо равенство

$$
\int_{-\infty}^{\infty} \int_{\Omega_{t}} \varphi\left\|F^{\prime}\right\|^{-1} d \mu_{\Omega_{t}} d t=\int_{V} \varphi d \mu
$$

Доказательству теоремы предпошлем ряд вспомогательных утверждений. Сразу отметим, что теорему достаточно доказать для случая $\mu \geqslant 0$; это и предполагается до конца параграффа.

Лемма 6. Пусть $W_{t}=\left\{\omega \in \Omega_{t}: F^{\prime}(\omega)=0\right\}$. Тогда почти при всех $t$

$$
\mu_{\Omega_{t}}\left(W_{t}\right)=0
$$


ДоказАТЕЛЬСТво. Пусть $\mu_{\Omega_{t}}\left(W_{t}\right)>0$ для некоторого $t$. Тогда существует поверхность $Q \in G(X, A, a, f)$ такая, что $Q \subset W_{t}, \mu_{\Omega_{t}}(Q)>0$. Отсюда

$$
\mu_{a}(Q)=\delta>0
$$

Для $\Delta t>0, \omega \in Q$ обозначим через $s_{0}=s_{0}(\omega, \Delta t)$ минимальньй из положительных корней уравнения $F(\omega+s a)=t+\Delta t$ (если указанных корней нет, то полагаем $\left.s_{0}=\infty\right)$. Легко видеть, что при фиксированном $\Delta t$ функция $s_{0}(\cdot, \Delta t)$ полунепрерывна снизу на $Q$ и, следовательно, борелевская. В силу дифференцируемости $F$ по направлению $a$ имеем для любого $\omega \in Q$

$$
F\left(\omega+s_{0} a\right)=F(\omega)+s_{0} D_{a} F(\omega)+o\left(s_{0}\right) .
$$

Учитывая, что $F(\omega)=t, D_{a} F(\omega)=0$, получаем

$$
\lim _{\Delta t \rightarrow 0} \frac{s_{0}(\omega, \Delta t)}{\Delta t}=\infty
$$

Для $c>0$ и последовательности $\Delta t_{n} \searrow 0$ положим $Q_{n}=\{\omega \in Q: \forall m \geqslant n$ $\left.c \Delta t_{m}<s_{0}\left(\omega, \Delta t_{m}\right)\right\}$; из $(22)$ и $(21)$ вытекает сушествование $N$ такого, что $\mu_{a}\left(Q_{N}\right)>\delta / 2$. Для $n \geqslant N$ положим

$$
Z_{n}=\bigcup_{\lambda \in\left[0, c \Delta t_{n}\right]}\left\{Q_{N}+\lambda a\right\}=T\left(Q_{N}+c \Delta t_{n} a\right) \backslash T\left(Q_{N}\right)
$$

(множества $T(Q)$ определены в начале $\S 1$ ). Из определений $s_{0}, Q_{n}$ вытекает, что $Z_{n} \subset V_{t+\Delta t_{n}} \backslash V_{t}$. Теперь для функции $u: R^{1} \rightarrow R^{1}, u(t)=\mu\left(V_{t}\right)$ имеем

$$
\begin{aligned}
\varlimsup_{n \rightarrow \infty}\left(\Delta t_{n}\right)^{-1}\left[u\left(t+\Delta t_{n}\right)-u(t)\right] & \geqslant \varlimsup_{n \rightarrow \infty}\left(\Delta t_{n}\right)^{-1} \mu\left(Z_{n}\right) \\
& =c D_{a} \mu\left[T\left(Q_{N}\right)\right]=c \mu_{a}\left(Q_{N}\right) \geqslant c \frac{\delta}{2},
\end{aligned}
$$

откуда в силу произвольности $c$ заключаем, что функция $u$ в точке $t$ имеет производное число, равное $\infty$. Но $u$ - монотонная ограниченная функция, так что почти при всех $t$ вьполняется неравенство $\mu^{\prime}(t)<\infty$. Лемма доказана.

Из доказанной леммы и условия $\mu\left\{z \in V: F^{\prime}(z)=0\right\}=0$ получаем, что теорему 5 достаточно доказать с заменой $V$ на (открытое же) множество $V \backslash\left\{F^{\prime}=0\right\}$ (и соответственно переопределенными $V_{t}, \Omega_{t}$ ). Учитывая это, далее считаем, что $F^{\prime}$ не обрашается в нуль на $V$ :

Введем функцию $\rho: R^{1} \times \Sigma_{V} \rightarrow[0, \infty]$,

$$
\rho(t, E)=\int_{E \cap \Omega_{t}}\left\|F^{\prime}\right\|^{-1} d \mu_{\Omega_{t}} .
$$

ЛЕмма 7. Функция $\nu: \Sigma_{V} \rightarrow[0, \infty], \nu(E)=\int_{-\infty}^{\infty} \rho(t, E) d t$, корректно определена, и имеет место равенство $\nu=\mu$. 
ДокАЗАТЕЛЬСтво. Пусть $z_{0} \in V, F\left(z_{0}\right)=t_{0}, a \in Z_{0},\left(F^{\prime}\left(z_{0}\right), a\right)>0,\|a\|=1$, $X$ - некоторое топологическое дополнение $R_{a}$ до $Z, x_{0}=P_{X}^{a} z_{0}, y_{0}=z_{0}-x_{0} \in R_{a}$. По теореме о неявной функции сушествуют интервал $\left(t_{1}, t_{2}\right) \ni t$, открытые (в $X$ и $R_{a}$ ) окрестности $U\left(x_{0}\right), U\left(y_{0}\right)$ точек $x_{0}, y_{0}$ и непрерьвная, непрерьвно дифференцируемая вдоль пространства $R^{1} \times\left[X \cap Z_{0}\right]$ функция $f:\left[t_{1}, t_{2}\right] \times U\left(x_{0}\right) \rightarrow U\left(y_{0}\right)$ такие, что для $t, x, y$ из указанных окрестностей эквивалентны равенства $F(x, y)=t$ и $y=f(t, x)$. Уменњшая при необходимости окрестности $\left(t_{1}, t_{2}\right), U\left(x_{0}\right)$, добьемся того, чтобы при $t \in\left(t_{1}, t_{2}\right), x \in U\left(x_{0}\right)$ выполнялись неравенства

$$
0<c_{1} \leqslant \frac{\partial f}{\partial t}(t, x)=\left(F^{\prime}(x+f(t, x)), a\right)^{-1} \leqslant c_{2}<\infty .
$$

Тогда множество $U\left(z_{0}\right)=\left\{z=x+y \in V: x \in U\left(x_{0}\right), f\left(t_{1}, x\right)<y<f\left(t_{2}, x\right)\right\}$ есть окрестность точки $z_{0}$ и для $t \in\left(t_{1}, t_{2}\right)$ выполняется равенство

$$
\Omega_{t} \cap U\left(z_{0}\right)=\operatorname{gr} f(t, \cdot) .
$$

Для множества $Q \in \Sigma_{U\left(x_{0}\right)}$ и чисел $r, s \in\left(t_{1}, t_{2}\right), r<s$, положим $V_{r, s}=\left(V_{s} \backslash V_{r}\right) \cap$ $\left(Q+R_{a}\right) \cap U\left(z_{0}\right)$. В силу леммы 4 (с естественными заменами и учетом $\left.(23)\right)$ при любом $t \in(r, s)$ справедливы равенства

$$
\begin{aligned}
\frac{d}{d t} \mu\left(V_{r, t}\right) & =\int_{\operatorname{gr} f(t, \cdot) \cap V_{r, s}} \frac{\partial f}{\partial t}\left(t, P_{X}^{a} \omega\right) d \mu_{a}^{t}(\omega)=\int_{\operatorname{gr} f(t, \cdot) \cap V_{r, s}}\left\|F^{\prime}\right\|^{-1} d \mu_{\Omega_{t}} \\
& =\int_{\Omega_{t} \cap V_{r, s}}\left\|F^{\prime}\right\|^{-1} d \mu_{\Omega_{t}}=\rho\left(t, V_{r, s}\right)
\end{aligned}
$$

(здесь $\left.\mu_{a}^{t}: \Sigma_{\mathrm{gr} f(t, \cdot)} \rightarrow R^{1}, \mu_{a}^{t}(W)=D_{a} \mu\left\{\bigcup_{\lambda \leqslant 0}(W+\lambda a)\right\}\right)$. В силу (23) второй член в (24) ограничен по $t$. Тогда функция $\left\{t \mapsto \mu\left(V_{r, t}\right)\right\}$ абсолютно непрерывна на $(r, s)$. Вне отрезка $[r, s]$ функция $\left\{t \mapsto \rho\left(t, V_{r, s}\right)\right\}$, очевидно, равна нулю. Отсюда и из (24) делаем два вывода: функция

$$
R^{1} \rightarrow R^{1}: t \mapsto \rho\left(t, V_{r, s}\right)
$$

борелевская; справедливо равенство

$$
\mu\left(V_{r, s}\right)=\int_{r}^{s} \rho\left(t, V_{r, s}\right) d t=\int_{-\infty}^{\infty} \rho\left(t, V_{r, s}\right) d t=\nu\left(V_{r, s}\right) .
$$

В силу (23) полукольцо $\left\{V_{r, s}\right\}$ порождает $\sigma$-алгебру $\Sigma_{U\left(z_{0}\right)}$. Теперь, учитывая сепарабельность $U\left(z_{0}\right)$ и счетную аддитивность функции $\rho$ по второму аргументу, из (25) получаем борелевость функции $R^{1} \rightarrow R^{1}: t \mapsto \rho(t, E)$ для любого $E \in \Sigma_{U\left(z_{0}\right)}$ и счетную аддитивность функции $\nu$ на $\Sigma_{U\left(z_{0}\right)}$, а из $(26)-$ равенство $\mu=\nu$ на $\Sigma_{U\left(z_{0}\right)}$. Но множества $U\left(z_{0}\right)$ образуют базу топологии пространства $V$, замкнутую относительно пересечений. Отсюда и из сепарабельности $V$ последовательно получаем борелевость функции $R^{1} \rightarrow R^{1}: t \mapsto \rho(t, E)$ для любого $E \in \Sigma_{V}$, счетную аддитивность функции $\nu$ и равенство $\mu=\nu$ на $V$. Лемма доказана.

ДокАЗАТЕЛЬСТво ТЕОРЕмЫ 5 . Так как $\varphi$ является $\mu$-интегрируемой, то $\varphi$ также $\nu$-интегрируема (по лемме 7). По обобщенной теореме Фубини для переходных мер [30; гл. III, п. 2] и лемме 7 теперь имеем

$$
\int_{-\infty}^{\infty} \int_{\Omega_{t}} \varphi\left\|F^{\prime}\right\|^{-1} d \mu_{\Omega_{t}} d t=\int_{-\infty}^{\infty} \int_{V} \varphi(z) \rho(t, d z) d t=\int_{V} \varphi d \nu=\int_{V} \varphi d \mu,
$$

что и требовалось. 


\section{§ 3. Формула интегрирования по частям}

Будем обозначать через $V^{0}, \bar{V}, \partial V$ внутренность, замыкание и границу множества $V \in \Sigma_{Z}$ соответственно. Точку $\omega_{0} \in \partial V$ назовем регулярной, если существует открытая окрестность $U\left(\omega_{0}\right) \ni \omega_{0}$ такая, что множество $\partial V \cap U\left(\omega_{0}\right)$ имеет вид $G(X, A, a, f)$; совокупность всех регулярных точек обозначим через $\Omega_{r}$ и положим $\Omega_{0}=\partial V \backslash \Omega_{r}$.

Ниже $a \in Z_{0},\|a\|=1, X$ - некоторое топологическое дополнение $R_{a}$ до $Z$, $\mu \in M^{1}, \Omega_{a}=\left\{\omega \in \Omega_{r}:(n(\omega), a)=0\right\}$. Через $\Omega_{n}$ обозначим множество всех точек $\omega \in \Omega_{r}$, в которых сушествует внешняя (по отношению к $V$ ) единичная нормаль $n(\omega) \in Z_{0}^{*}$ (нормаль $n(\omega)$ называется внешней, если для любого вектора $b \in Z_{0}$, удовлетворяюшего условию $(n(\omega), b)>0$, существует $\varepsilon=\varepsilon(b) \geq 0$ такое, что при всех $\delta \in(0, \varepsilon)$ выполняются включения $\left.\omega-\delta b \in V^{0}, \omega+\delta b \in Z \backslash \bar{V}\right)$. Далее функция $n$ считается равной нулю на множестве $\partial V \backslash \Omega_{n}$ (поскольку множество $\partial V \backslash \Omega_{n}$ замкнуто в $\partial V$, то функция $n-$ борелевская).

Теорема 6 (формула интегрирования по частям). Пусть множсество $\partial \mathrm{V}$ есть поверхность, функиия $u: \bar{V} \rightarrow R^{1}$ непрерывна, ограничена, на $V^{0}$ имеет непрерывную ограниченную производную по направлению а и такова, что

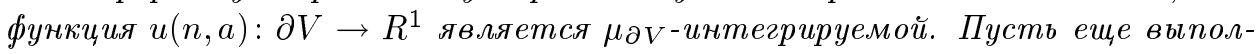
нено условие

$$
|\mu|\left(\Omega_{0}+R_{a}\right)=0 .
$$

Тогда справедливо равенство

$$
\int_{V} D_{a} u d \mu+\int_{V} u d D_{a} \mu=\int_{\partial V} u(n, a) d \mu_{\partial V} .
$$

ДокАЗАТЕЛЬСТво. Считаем, что $\mu \geqslant 0$. Вспоминая определение функций $\varphi, \psi$ (см. $\S 1$ ), видим, что на $V^{0}$ верно равенство

$$
\left(D_{a} u\right) \mu+u D_{a} \mu=\left[D_{a}(u \varphi)\right]\left(\mu_{X} \times l\right),
$$

и по теореме Фубини

$$
\begin{aligned}
\int_{V^{0}} D_{a} u d \mu+\int_{V^{0}} u d D_{a} \mu & =\int_{V^{0}} D_{a}(u \varphi) d\left(\mu_{X} \times l\right) \\
& =\int_{X} \int_{S_{x}^{a}\left(V^{0}\right)} D_{a}(u \varphi) d l d \mu_{X}(x) \\
& =\int_{X} \sum_{m=1}^{\infty}\left[u \varphi\left(x, t_{m}^{x}\right)-u \varphi\left(x, s_{m}^{x}\right)\right] d \mu_{X}(x),
\end{aligned}
$$

где $\left(s_{m}^{x}, t_{m}^{x}\right)$ есть составляющие интервалы открытого множества $S_{x}^{a}\left(V^{0}\right)$. Отметим, что из предположений относительно $u$ вытекает сушествование всех интегралов в (29); более того, фигурирующий в правой части ряд сходится абсолютно.

$\mathrm{B}$ [31] доказано включение $\Omega_{a}+R_{a} \subset \Sigma_{Z}$ и равенство $\mu\left(\Omega_{a}+R_{a}\right)=0$. (Доказательство проводилось для банахова $Z$, однако банахова структура не использовалась, и доказательство проходит для сепарабельного пространства Фреше.) 
Отсюда и из (27) получаем включение $X_{0}=P_{X}^{a}\left(\Omega_{0} \cup \Omega_{a}\right) \subset \Sigma_{X}$ и равенство $\mu_{X}\left(X_{0}\right)=0\left(P_{X}^{a}\right.$ есть проектор $Z$ на $X$, параллельный $\left.a\right)$. Обозначим через $\delta_{y}$ меру Дирака на $R_{a}$, сосредоточенную в точке $y \in R_{a}$, и рассмотрим функцию $\rho:\left(X \backslash X_{0}\right) \times \Sigma_{R_{a}} \rightarrow[0, \infty]$ такую, что

$$
\rho(x, E)=\sum_{y:(x, y) \in \Omega_{n}}|(u \varphi)(x, y)| \delta_{y}(E)
$$

(ряд не более чем счетен). Из определения множеств $\Omega_{r}, \Omega_{a}, X_{0}$ вытекает, что если $x \in X \backslash X_{0}, z=x+y \in \partial V$, то сушествуют открытые в пространствах $X$, $R_{a}$ окрестности $U(x), U(y)$ точек $x, y$ и функция $f: U(x) \rightarrow R_{a}$ такие, что

$$
\partial V \cap[U(x) \times U(y)]=G(X, U(x), a, f) .
$$

Отсюда, из определения поверхности и сепарабельности $\Omega_{n}$ получаем существование простых непересекающихся поверхностей $Q_{m} \subset G\left(X, A_{m}, a, f_{m}\right)$, $m=1,2, \ldots$, таких, что $\Omega_{n} \backslash\left(X_{0}+R_{a}\right)=\bigcup_{m} Q_{m}$. Положим $\rho_{m}:\left(X \backslash X_{0}\right) \times \Sigma_{R_{a}}$ $\rightarrow[0, \infty]$,

$$
\rho_{m}(x, E)=\mathbf{1}_{Q_{m}}\left(x, f_{m}(x)\right)\left|u \varphi\left(x, f_{m}(x)\right)\right| \delta_{f_{m}(x)}(E) .
$$

Так как функция $g_{m, E}: Q_{m} \rightarrow[0, \infty]$,

$$
g_{m, E}:(x, y) \mapsto|u \varphi(x, y)| \mathbf{1}_{(X+E) \cap Q_{m}}(x, y)
$$

борелевская, а функция $P_{X}^{a}\left(Q_{m}\right) \rightarrow Q_{m}: x \mapsto\left(x, f_{m}(x)\right)$ есть метрический изоморфизм пространств $P_{X}^{a}\left(Q_{m}\right)$ и $Q_{m}$, то функция $\rho_{m}(x, E)=g_{m, E}\left(x, f_{m}(x)\right)$ борелевская по первому аргументу. Но тогда и $\rho=\sum_{m} \rho_{m}$ борелевская по первому аргументу. Так как $\rho$, очевидно, счетноаддитивна по второму аргументу, то $\rho$ есть ([0, $\infty]$-значная) переходная мера из $X \backslash X_{0}$ в $R_{a}$, и мы вправе рассмотреть меру $\nu: \Sigma_{X \backslash X_{0}} \times \Sigma_{R_{a}} \rightarrow[0, \infty]$,

$$
\nu(B)=\int_{X \backslash X_{0}} \rho\left(x, S_{x}^{a}(B)\right) d \mu_{X}(x) .
$$

Положим $\nu_{1}=|u(n, a)| \mu_{\partial V}$ и продолжим меру $\nu_{1}: \Sigma_{\partial V} \rightarrow R^{1}$ на $\Sigma_{Z}$, полагая $\nu_{1}(B)=\nu_{1}(B \cap \partial V)$. Сейчас наша цель - доказательство равенства

$$
\nu\left[\left(X \backslash X_{0}\right)+R_{a}\right]=\nu_{1}\left[\left(X \backslash X_{0}\right)+R_{a}\right] .
$$

Возьмем точку $z=x+y, x \in X \backslash X_{0}, y \in R_{a}$, и рассмотрим три случая.

1. $z \in \Omega_{n}$. Выберем окрестности $U(x), U(y)$, фигурирующие в $(30)$, и пусть $U_{1} \subset U(x), U_{2} \subset U(y)$ есть открытые множества. Положим $W=\partial V \cap\left[\left(U_{1} \cap\left(X \backslash X_{0}\right)\right) \times U_{2}\right]$. Учитывая определения мер $\nu, \nu_{1}$, равенство (30) и применяя лемму 3 , получим

$$
\begin{aligned}
\nu\left[\left(U_{1} \cap\left(X \backslash X_{0}\right)\right) \times U_{2}\right] & =\int_{P_{X}^{a} W}|u \varphi|(x, f(x)) d \mu_{X}(x)=\int_{W}|u| d \mu_{a} \\
& =\int_{W}|u(n, a)| d \mu_{\partial V}=\nu_{1}\left[\left(U_{1} \cap\left(X \backslash X_{0}\right)\right) \times U_{2}\right] .
\end{aligned}
$$


2. $z \in \Omega_{r} \backslash \Omega_{n}$. Тогда в формуле (30) окрестности $U(x), U(y)$ можно взять столь мальми, что $\partial V \cap[U(x) \times U(y)] \subset \Omega_{r} \backslash \Omega_{n}$, так что для любых открытых множеств $U_{1} \subset U(x), U_{2} \subset U(y)$ правая часть в $(32)$ есть нуль (напомним, что $n=0$ на $\left.\partial V \backslash \Omega_{n}\right)$. Левая часть в (32) также есть нуль, ибо $[U(x) \times U(y)] \cap \Omega_{n}=\varnothing$.

3. $z \notin \partial V$. В силу замкнутости $\partial V$ сушествуют окрестности $U(x), U(y)$ точек $x, y$ такие, что $[U(x) \times U(y)] \cap \partial V=\varnothing$, так что для любых открытых множеств $U_{1} \subset U(x), U_{2} \subset U(y)$ крайние члены в (32) обрашаются в нуль.

Итак, для любой точки $z$ пространства $\left(X \backslash X_{0}\right)+R_{a}$ сушествует открытый (в $\left.\left(X \backslash X_{0}\right)+R_{a}\right)$ прямоугольник $U(z)=\left[\left(U(x) \cap\left(X \backslash X_{0}\right)\right) \times U(y)\right] \ni z$ такой, что для любого открытого (в $\left.\left(X \backslash X_{0}\right)+R_{a}\right)$ прямоугольника $U_{1} \times U_{2} \subset U(z)$ вьполняется равенство $\nu_{1}\left(U_{1} \times U_{2}\right)=\nu\left(U_{1} \times U_{2}\right)$. Отсюда, из счетной аддитивности мер $\nu, \nu_{1}$ и сепарабельности $\left(X \backslash X_{0}\right)+R_{a}$ вытекает $(31)$.

Из (31), конечности меры $\nu_{1}$ (предположение теоремы) и определения меры $\nu$ вытекает, что для $\mu_{X}$-почти всех $x \in X \backslash X_{0}$ мера $\rho(x, \cdot)$ конечна; после переобозначений будем считать, что мера $\rho(x, \cdot)$ конечна для всех $x \in X \backslash X_{0}$. Но тогда корректно определена функция $\rho_{0}:\left(X \backslash X_{0}\right) \times \Sigma_{R_{a}} \rightarrow R^{1}$,

$$
\rho_{0}(x, E)=\sum_{y:(x, y) \in \Omega_{n}}[\operatorname{sign}(n(x+y), a)](u \varphi)(x, y) \delta_{y}(E)
$$

очевидно, эта функция счетноаддитивна по второму аргументу. Легко видеть, что для всех $x \in X \backslash X_{0}$ справедливо равенство

$$
\rho_{0}\left(x, R^{a}\right)=\sum_{m=1}^{\infty}\left[u \varphi\left(x, t_{m}^{x}\right)-u \varphi\left(x, s_{m}^{x}\right)\right]
$$

(см. (29)). Действительно, в силу абсолютной сходимости ряда в правой части (34) его сумма не зависит от перестановки членов. Если точка $z=(x, y) \in \Omega_{r} \backslash \Omega_{n}$ такова, что обе нормали $\pm n(z)$ являются внутренними относительно $V$, то для некоторых $m, k$ верно $t_{m}^{x}=s_{k}^{x}$ и соответствующие члены в правой части (34) взаимно уничтожаются. За указанным исключением ряды (33), (34) перестановочно совпадают.

Аналогично, для любого интервала $I=(\alpha, \beta) \subset R_{a}$ имеем

$$
\begin{aligned}
\rho_{0}(x, I)= & \rho_{0}\left(x, S_{x}^{a}\left(V^{0} \cap(X+I)\right)\right)=\sum_{m=1}^{\infty}\left[u \varphi\left(x, \bar{t}_{m}^{x}\right)-u \varphi\left(x, \bar{s}_{m}^{x}\right)\right] \\
& +\mathbf{1}_{V(\alpha)}(x)(u \varphi)(x, \alpha)-\mathbf{1}_{V(\beta)}(x)(u \varphi)(x, \beta),
\end{aligned}
$$

где $\left(\bar{s}_{m}^{x}, \bar{t}_{m}^{x}\right)$ есть составляющие интервалы множества $S_{x}^{a}\left(V^{0} \cap(X+I)\right), V(\gamma)=$ $P_{X}^{a}[\bar{V} \cap(X+\gamma)], \gamma \in R_{a}, \mathbf{1}_{V(\gamma)}$ - характеристическая функция множества $V(\gamma)$. Записывая равенство (29) с заменой $V^{0}$ на $V^{0} \cap(X+I)$, видим, что функция $x \mapsto \sum_{m=1}^{\infty}\left[u \varphi\left(x, \bar{t}_{m}^{x}\right)-u \varphi\left(x, \bar{s}_{m}^{x}\right)\right]$ является $\Sigma_{X}$-измеримой, так что с учетом $(35)$ функция $x \mapsto \rho_{0}(x, I)$ является $\Sigma_{X \backslash X_{0}}$-измеримой. 
Итак, функция $\rho_{0}$ есть переходная мера. Поскольку для любого $x \in X \backslash X_{0}$ имеет место равенство $\left|\rho_{0}(x, \cdot)\right|\left(R_{a}\right)=\rho\left(x, R_{a}\right)$, а функция $x \mapsto \rho\left(x, R_{a}\right)$ является $\mu_{X}$-интегрируемой (равенство (31)), то [26] корректно определена мера $\nu_{0}: \Sigma_{X \backslash X_{0}} \times \Sigma_{R_{a}} \rightarrow R^{1}$,

$$
\nu_{0}(B)=\int_{X \backslash X_{0}} \rho_{0}\left(x, S_{x}^{a}(B)\right) d \mu_{X}(x),
$$

см. [26]. Учитывая определение внешней нормали и проводя те же рассуждения, что и при доказательстве равенства (31), получим равенство

$$
\nu_{0}\left[\left(X \backslash X_{0}\right)+R_{a}\right]=u(n, a) \mu_{\partial V}\left[\left(X \backslash X_{0}\right)+R_{a}\right] .
$$

Левая часть (37) с учетом (36) (здесь $B=X \backslash X_{0}+R_{a}$ ), (34), (29) и равенства $\mu_{X}\left(X_{0}\right)=0$ равна левой части (29). Правая часть (37) равна правой части (28). Действительно,

$$
\begin{aligned}
u(n, a) \mu_{\partial V}( & \left.X_{0}+R_{a}\right)=u(n, a) \mu_{\partial V}\left[\left(\Omega_{r} \backslash \Omega_{a}\right) \cap\left(X_{0}+R_{a}\right)\right] \\
& +u(n, a) \mu_{\partial V}\left[\Omega_{a} \cap\left(X_{0}+R_{a}\right)\right]+u(n, a) \mu_{\partial V}\left[\Omega_{0} \cap\left(X_{0}+R_{a}\right)\right] .
\end{aligned}
$$

Как уже указывалось выше, мера $u(n, a) \mu_{\partial V}$ равна нулю локально на $\left(\Omega_{r} \backslash \Omega_{a}\right) \cap$ $\left(X_{0}+R_{a}\right)$ и, следовательно, $u(n, a) \mu_{\partial V}\left[\left(\Omega_{r} \backslash \Omega_{a}\right) \cap\left(X_{0}+R_{a}\right)\right]=0$. Два оставшшхся слагаемых равны нулю, так как $(n, a)=0$. Теперь остается только заметить, что $u(n, a) \mu_{\partial V}(Z)=u(n, a) \mu_{\partial V}(\partial V)$.

Итак,

$$
\int_{V^{0}} D_{a} u d \mu+\int_{V^{0}} u d D_{a} \mu=\int_{\partial V} u(n, a) d \mu_{\partial V} .
$$

Так как $\partial V$ есть поверхность, а мера $\mu$ дифференцируема по направлениям из $Z_{0}$, то $\mu=0$ локально на $\partial V$ и, значит, $\mu(\partial V)=0$. Отсюда и $\left|D_{a} \mu\right|(\partial V)=0$ (так как $\left.D_{a} \mu \ll \mu\right)$. Таким образом, левая часть (38) равна левой части (28), и теорема доказана.

ЗАмечаниЕ. Теорема справедлива и при замене условия (25) условием $\left|\mu_{\partial V}\right|\left(\Omega_{0}\right)=0 ;$ доказательство, однако, усложняется.

\section{§4. Формулы Гаусса-Остроградского и Грина}

В оставшейся части работы $Z_{0}=H$ есть сепарабельное гильбертово пространство, $\left\{e_{j}\right\}$ - ортонормированный базис в $H, L(H), L_{1}(H)$ - соответственно пространства линейных непрерывных и ядерных операторов из $H$ в $H$. Предполагается, что $L(H)$ наделено равномерной нормой, $L_{1}(H)$ - ядерной нормой $\|\cdot\|_{1}$. Используются обозначения: $C^{n}(V, Y)$ - пространство непрерывных ограниченных функций, определенных на открытом множестве $V \subset Z$, принимающих значения в нормированном пространстве $Y$ и $n$ раз непрерывно и ограниченно дифференцируемых вдоль пространства $H ; C^{n}(\bar{V}, Y)$ - подпространство функций из $C^{n}\left(V^{0}, Y\right)$, производные которых порядка $0, \ldots, n-1$ допускают непрерывное продолжение на множество $\bar{V}(V \subset Z)$.

Назовем множество $V \in \Sigma_{Z} \mu$-нормальныц, если: 
1) $\partial V$ есть поверхность;

2 ) для любого $j=1,2, \ldots$ равенство (25) выполняется с заменой $a$ на $e_{j}$.

Далее до конца параграфа $V$ есть $\mu$-нормальное множество.

Пусть функция $b \in C^{1}(\bar{V}, H)$ такова, что $b^{\prime}(z) \in L_{1}(H)$ при любом $z \in V^{0}$. Тогда корректно определена функция $\operatorname{div} b: V^{0} \rightarrow R^{1}: z \mapsto \operatorname{Tr} b^{\prime}(z)$. Положим по определению

$$
\int_{V} b d \mu^{\prime}=\sum_{j=1}^{\infty} \int_{V}\left(b, e_{j}\right) d D_{e_{j}} \mu
$$

если ряд справа сходится.

Теорема 7 (формула Гаусса-Остроградского). Пусть функция $V^{0} \rightarrow R^{1}$ : $z \mapsto\left\|b^{\prime}(z)\right\|_{1}$ является $\mu$-интегрируемой, функиия $\|b\|: \partial V \rightarrow R^{1}$ является

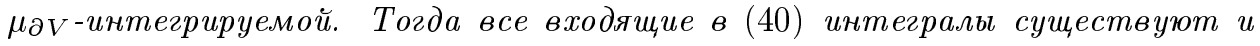
справедливо равенство

$$
\int_{V} \operatorname{div} b d \mu+\int_{V} b d \mu^{\prime}=\int_{\partial V}(b, n) d \mu_{\partial V}
$$

ДокАЗАТЕЛЬСТво. Полагая в $(28) a=e_{j}, u=\left(b, e_{j}\right)$ и складывая равенства по $j=1, \ldots, N$, получим

$$
\int_{V} \sum_{j=1}^{N}\left(b^{\prime} e_{j}, e_{j}\right) d \mu+\sum_{j=1}^{N} \int_{V}\left(b, e_{j}\right) d D_{e_{j}} \mu=\int_{\partial V} \sum_{j=1}^{N}\left(b, e_{j}\right)\left(n, e_{j}\right) d \mu_{\partial V}
$$

В силу соотношения $\left|\sum_{j=1}^{N}\left(b^{\prime}(z) e_{j}, e_{j}\right)\right| \leqslant\left\|b^{\prime}(z)\right\|_{1}[32 ;$ гл. VI, п. 6], неравенства Коши-Буняковского, предположений теоремы 7 и теоремы Лебега законен переход к пределу под знаком левого и правого интегралов. Отсюда вытекает сушествование среднего интеграла в (40) и само равенство (40). Теорема доказана.

Пусть $A \in C^{1}(\bar{V}, L(H)), b \in C^{1}(\bar{V}, H), u \in C^{2}\left(\bar{V}, R^{1}\right), \alpha \in C^{0}\left(V^{0}, R^{1}\right)$ таковы, что для любого $z \in V^{0}$ выполнены включения

$$
\begin{gathered}
\left(A^{*}(z) u^{\prime}(z)\right)^{\prime} \in L_{1}(H), \quad(u b)^{\prime}(z) \in L_{1}(H) ; \\
L^{*} u: V^{0} \rightarrow R^{1}: z \mapsto \operatorname{div}\left[A^{*}(z) u^{\prime}(z)\right]-\operatorname{div}(u b)(z)+\alpha(z) u(z) .
\end{gathered}
$$

Теорема 8 (первая формула Грина). Пусть функция $V^{0} \rightarrow R^{1}: z \mapsto$ $\left\|\left(A^{*}(z) u^{\prime}(z)\right)^{\prime}\right\|_{1}$ является $\mu$-интегрируемой, функиия $\left\|A^{*} u^{\prime}\right\|$ является

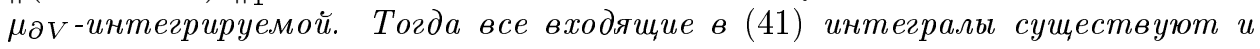
справедливо равенство

$$
\int_{V}\left[L^{*} u+\operatorname{div} u b-\alpha u\right] d \mu+\int_{V}\left(A^{*} u^{\prime}\right) d \mu^{\prime}=\int_{\partial V} D_{A n} u d \mu_{\partial V} .
$$

Доказательство получается из (40) с заменой $b$ на $A^{*} u^{\prime}$.

Запись второй формулы Грина сложнее; перейдем к соответствуюшим формулировкам. Далее предполагается, что мера $\mu$ дважды дифференцируема по $H$. 
Заметим, что функция $a_{i j}: \bar{V} \rightarrow R^{1}: z \mapsto\left(e_{i}, A(z) e_{j}\right)(\forall i, j)$ принадлежит пространству $C^{1}\left(\bar{V}, R^{1}\right)$ и, следовательно, повторньй ряд в правой части формального равенства

$$
\int_{V} u d\left(\operatorname{div} A \mu^{\prime}\right)=\sum_{j=1}^{\infty} \sum_{i=1}^{\infty}\left[\int_{V} u D_{e_{i}} a_{i j} d D_{e_{j}} \mu+\int_{V} u a_{i j} d D_{e_{i} e_{j}}^{2} \mu\right]
$$

корректно определен. Если этот ряд сходится и сходится ряд (39) с заменой $b$ на $u b$, то положим по определению

$$
\int_{V} u d L \mu=\int_{V} u d\left(\operatorname{div} A \mu^{\prime}\right)+\int_{V} u b d \mu^{\prime}+\int_{V} \alpha d \mu .
$$

Пусть для любого $j=1,2, \ldots$ функция $u\left(A^{*} n, e_{j}\right): \partial V \rightarrow R^{1}$ является $\left(D_{e_{j}} \mu\right)_{\partial V^{-}}$ интегрируемой. Положим по определению

$$
\int_{\partial V} u d D_{A^{*} n} \mu_{\partial V}=\sum_{j=1}^{\infty} \int_{\partial V} u\left(A^{*} n, e_{j}\right) d\left(D_{e_{j}} \mu\right)_{\partial V}
$$

если ряд справа сходится.

ТЕорема 9 (вторая формула Грина). Предположим, что функиии $\left\|u^{\prime \prime}(z)\right\|_{1}$, $\left\|(u b)^{\prime}(z)\right\|_{1},\left\|\left(A^{*} u^{\prime}\right)^{\prime}(z)\right\|_{1}$ являются $\mu$-интегрируемвцми, функции $\left\|u^{\prime}(z)\right\|$,

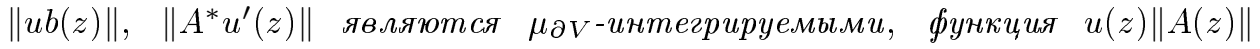


хотя бъ один из рядов (42), (43), то все входящие в (44) интеграль существуют и справедливо равенство

$$
\int_{V} L^{*} u d \mu-\int_{V} u d L \mu=\int_{\partial V}\left[D_{A n} u-u(b, n)\right] d \mu_{\partial V}-\int_{\partial V} u d D_{A^{*} n} \mu_{\partial V}
$$

ДокАЗАТЕльство. Заметим сначала, что соотношение $a_{i j} D_{e_{j}} \mu \ll \mu$ влечет вьполнение (27) с заменой $|\mu|_{X}$ на $\left|a_{i j} D_{e_{j}} \mu\right|_{X}$. Пользуясь теперь теоремой 6 , заменяя в (28) $a$ на $e_{i}, \mu$ на $a_{i j} D_{e_{j}} \mu$ и суммируя по индексам $i, j$, получим

$$
\begin{array}{r}
\sum_{j=1}^{k} \sum_{i=1}^{m} \int_{V} a_{i j} D_{e_{j}} u d D_{e_{j}} \mu+\left[\sum_{j=1}^{k} \sum_{i=1}^{m}\left(\int_{V} u D_{e_{i}} a_{i j} d D_{e_{j}} \mu+\int_{V} u a_{i j} d D_{e_{i} e_{j}}^{2} \mu\right)\right] \\
=\sum_{j=1}^{k} \sum_{i=1}^{m} \int_{\partial V} u\left(n, e_{i}\right) a_{i j} d\left(D_{e_{j}} \mu\right)_{\partial V} .
\end{array}
$$

Из условия на $A, u$ и теоремы Лебега находим

$$
\lim _{m \rightarrow \infty} \sum_{j=1}^{k} \sum_{i=1}^{m} \int_{V} a_{i j} D_{e_{i}} u d D_{e_{j}} \mu=\sum_{j=1}^{k} \int_{V}\left(A^{*} u^{\prime}, e_{j}\right) d D_{e_{j}} \mu .
$$


Из теоремы 7 теперь вытекает сушествование интеграла

$$
\int_{V} A^{*} u^{\prime} d \mu^{\prime}=\lim _{k \rightarrow \infty} \sum_{j=1}^{k} \int_{V}\left(A^{*} u^{\prime}, e_{j}\right) d D_{e_{j}} \mu .
$$

Из условия $\left(D_{e_{j}} \mu\right)_{\partial V}$-интегрируемости функции $u\|A\|$ и теоремы Лебега получаем равенство

$$
\lim _{m \rightarrow \infty} \sum_{j=1}^{k} \sum_{i=1}^{m} \int_{\partial V} u\left(n, e_{i}\right) a_{i j} d\left(D_{e_{j}} \mu\right)_{\partial V}=\sum_{j=1}^{k} u\left(A^{*} n, e_{j}\right) d\left(D_{e_{j}} \mu\right)_{\partial V} .
$$

Итак, всегда сушествует предел по $m$ выражения, стояшего в квадратных скобках в (45). Теперь из (47) и (46) получаем, что сходимость ряда (42) эквивалентна сходимости ряда (43). В случае наличия такой сходимости (45) и (46) дают равенство

$$
\int_{V} A^{*} u^{\prime} d \mu^{\prime}+\int_{V} u d\left(\operatorname{div} A \mu^{\prime}\right)=\int_{\partial V} u d D_{A^{*} n} \mu_{\partial V} .
$$

Теперь (44) вытекает из (41) и (40) (здесь нужно заменить $b$ на $u b)$. Теорема доказана.

ЗАмЕчАниЕ. Теоремы $7-9$ справедливы и при замене условия $\mu$-нормальности множества $V$ условием $|\mu|_{\partial V}\left(\Omega_{0}\right)=0$.

\section{Список литературы}

1. Kuо H. H. Integration theory on infinite-dimensional manifolds // Trans. Amer. Math. Soc. 1971. № 159. P. 57-78.

2. Goodman V. A givergence theorem for Hilbert space // Trans. Amer. Math. Soc. 1972. № 164. P. 411-426.

3. Го Х. С. Гауссовские меры в банаховых пространствах. М.: Мир, 1979.

4. Скороход А. В. Интегрирование в гильбертовом пространстве. М.: Наука, 1975.

5. Угланов А. В. Дифференциальнте уравнения второго порядка для функций бесконечномерного аргумента // Докл. АН СССР. 1976. Т. 230. № 1. С. 44-47.

6. Угланов А. В. Поверхностные интегралы в банаховом пространстве // Матем. сб. 1979. T. 110. № 2. C. 189-217.

7. Ефимова Е. И., Угланов А. В. Формулы векторного анализа на банаховом пространстве // Докл. АН СССР. 1983. Т. 271. №6. С. 1302-1307.

8. Далецкий Ю. Л., Марянин Б. Д. Гладкие меры на бесконечномерных многообразиях // Докл. АН СССР. 1985. Т. 285. №6. С. 1297-1300.

9. Смолянов О.Г. Потоки де Рама и формула Стокса в гильбертовом пространстве // Докл. АН СССР. 1986. Т. 286. №3. С. 554-558.

10. Норин Н. В. Стохастические интегралы и диффференцируемые меры // Теория вероятн. и ее прим. 1987. Т. 32. №1. С. 114-124.

11. Airault H., Malliavin P. Intégration géométrique sur l'espace de Wiener // Bull. Sci. Math. 1988. V. 112. № 1. P. 3-52.

12. Смородина Н. В. Формула Гаусса-Остроградского для пространства конфигураций // Теория вероятн. и ее прим. 1990. Т. 35. № 4. С. 727-739.

13. Bogachev V.I. Smooth measures, the Malliavin calculus and approximations in infinite-dimensional spaces // Acta Univ. Carolin. Math. Phys. 1990. V. 31. № 2. P. 9-23.

14. Угланов А.В. О сингулярных обобщенных функциях бесконечномерного аргумента // Матем. заметки. 1977. Т. 22. №4. С. 543-551. 
15. Угланов A. В. Поверхностные интегралы и дифференциальные уравнения на бесконечномерном пространстве // Докл. АН СССР. 1979. Т. 247. №6. С. 1331-1335.

16. Угланов А.В. О делении обобщенных функций бесконечного числа переменных на полиномы // Докл. АН СССР. 1982. Т. 264. № 5. С. 1096-1099.

17. Беляев A.A. Теорема о среднем для функций, гармонических в области гильбертова пространства // Вестн. МГУ. Сер. 1. Матем., мех. 1982. № 5. С. 32-35.

18. Норин Н. В. Тепловые потенциалы на гильбертовом пространстве // Матем. заметки. 1984. T. 35. № 4. C. 531-548.

19. Норин Н. В. Аналитичность бесконечномерных тепловых потенциалов // Матем. заметки. 1985. Т. 35. № 4. С. 365-374.

20. Угланов А. В. Интегральное представление функций на банаховых пространствах // Докл. АН СССР. 1985. Т. 292. № 4. С. 800-804.

21. Норин Н. В. Теорема о среднем, связанная с бесконечномернцми тепловьми потенциалами // Теория функций, функц. анализ и их прилож. 1987. № 48. С. 111-117.

22. Угланов A.B О гладкости распределений функционалов от случайных процессов // Теория вероятн. и ее прим. 1988. Т. 23. № 2. С. 535-544.

23. Угланов $A . B$. Формула Ньютона-Лейбница на банаховом пространстве и приближение функций бесконечномерного аргумента // Изв. АН СССР. 1987. Т. 51. № 1. С. 152-170.

24. Uglanov A. $V$. Integrals with respect to vector-valued measures: theoretical problems and applications // Amer. Math. Soc. Transl. Ser. 2. 1995. V. 163. P. 171-184.

25. Uglanov A. V. Calculus of variations on banach spaces // Internat. Conf. "Modern Banach Space Theory". Abstracts: Kent Univ. Publ., 1996.

26. Угланов А. В. Теорема Фубини для векторных мер // Матем. сб. 1990. Т. 181. № 3. C. $423-432$.

27. Угланов A. В. Вариационные задачи на абстрактном банаховом пространстве // Докл. АН. (в печати).

28. Далецкий Ю. Л., Фомин С. В. Меры и дифференциальные уравнения в бесконечномерных пространствах. М.: Наука, 1983.

29. Угланов A. В. Частное гладких мер есть гладкая функция // Изв. вузов. Сер. матем. 1989. №9. С. $72-76$.

30. Неве Ж. Математические основы теории вероятностей. М.: Мир, 1969.

31. Ефимова Е. И. О теореме Сарда на банаховом пространстве // Изв. вузов. Сер. матем. 1988. №9. C. 37-39.

32. Рид М., Саймон Б. Методы современной математической физики. Т. 1. М.: Мир, 1977.

Ярославский государственньй университет

Поступила в редакцию 